\title{
Proposed guidelines for the management of nodding syndrome
}

*Idro $\mathrm{R}^{1,2}$, Musubire K A ${ }^{3}$, Byamah Mutamba B ${ }^{4}$, Namusoke $\mathrm{H}^{1}$, Muron J4 ${ }^{4}$, Abbo $\mathrm{C}^{5}$, Oriyabuzu $\mathrm{R}^{5}$, Ssekyewa J , Okot $\mathrm{C}^{6}$, Mwaka $\mathrm{D}^{3}$, Ssebadduka $\mathrm{P}^{7}$, Makumbi I ${ }^{8}$, Opar $\mathrm{B}^{8}$, Aceng $\mathrm{JR}^{8}$, Mbonye $\mathrm{AK}^{8,9}$

1. Department of Paediatrics and Child Health, Mulago hospital/Makerere University College of Health Sciences, Kampala, Uganda

2. Centre for Tropical Medicine, Nuffield Department of Medicine, Oxford University, UK

3. Department of Internal Medicine, Mulago hospital/Makerere University College of Health Sciences, Kampala, Uganda

4. Butabika National Referral Mental Hospital, Kampala, Uganda

5. Department of Psychiatry, Mulago hospital/Makerere University College of Health Sciences, Kampala, Uganda

6. World Health Organization, Uganda Country Office, Kampala, Uganda

7. Department of Obstetrics and Gynaecology, Mulago hospital/Makerere University College of Health Sciences, Kampala, Uganda

8. Ministry of Health Headquarters, Kampala, Uganda

9. Department of Community Health \& Behavioral Sciences, School of Public Health, Makerere University College of Health Sciences, Kampala, Uganda

\begin{abstract}
Nodding Syndrome is a poorly understood neurologic disorder of unknown aetiology that affects children and adolescents in Africa. Recent studies have suggested that the head nods are due to atonic seizures and Nodding Syndrome may be classified as probably symptomatic generalised epilepsy. As part of the Ugandan Ministry of Health clinical management response, a multidisciplinary team developed a manual to guide the training of health workers with knowledge and skills to manage the patients. In the absence of a known cause, it was decided to offer symptomatic care. The objective is to relieve symptoms, offer primary and secondary prevention for disability and rehabilitation to improve function. Initial management focuses on the most urgent needs of the patient and the immediate family until 'stability' is achieved. The most important needs were considered as seizure control, management of behavioural and psychiatric difficulties, nursing care, nutritional and subsequently, physical and cognitive rehabilitation. This paper summarises the processes by which the proposed guidelines were developed and provides an outline of the specific treatments currently being provided for the patients. African Health Sciences 2013; 13(2): 219 - 232 http://dx.doi.org/10.4314/ahs.v13i2.4
\end{abstract}

\section{Introduction}

Nodding Syndrome is a poorly understood neurologic disorder of unknown aetiology that affects children and adolescents in parts of subSaharan Africa. There are probably between 5000 10,000 affected children in East Africa ${ }^{1}$. This phenomena was first reported in Tanzania in $1960{ }^{2}{ }^{2}$ and subsequent reports have come from Liberia ${ }^{3}$, South Sudan ${ }^{4,5}$ and Northern Uganda where there are several thousand affected individuals ${ }^{6,7}$. It however may have existed earlier in Uganda as Nakalanga syndrome ${ }^{8-11}$. The syndrome as seen in Northern Uganda and South Sudan is characterized by head nodding and complicated by a variable presence of other seizure types, cognitive and motor decline,

*Corresponding author:
Dr Richard Idro
Department of Paediatrics and Child Health
Makerere University College of Health Sciences
P.O Box 7072
Kampala, Uganda
Email: ridro1@gmail.com

African Health Sciences Vol 13 Issue 2 June 2013 wasting, stunting, behaviour and psychiatric difficulties. Five clinical stages with deteriorating seizures, neuro-cognitive, physical and psychiatric disability can be identified in Ugandan patients. These include a prodrome; development of head nodding and cognitive impairment; other seizure types; multiple complications and, severe disability. Recent studies have concluded that the head nods are due to atonic seizures ${ }^{12}$ and that this epidemic epilepsy syndrome may be classified as probably symptomatic generalised epilepsy (Richard Idro, unpublished). This paper is a summary of proposed guidelines for the management of Nodding Syndrome in Uganda.

\section{Case definitions}

The first International Scientific Meeting on Nodding Syndrome held in Kampala (Uganda) from $30^{\text {th }}$ July to $1^{\text {st }}$ August 2012 agreed on and adopted the following case definitions for Nodding Syndrome ${ }^{13}$ :

a) A suspected case: Is reported head nodding in a previously normal person; head nodding was 
defined as repetitive, involuntary drops of the head to the chest on two or more occasions. This case definition is used at the community level.

b) A probable case: Is a suspected case with:

* Both of the following major criteria:

$>$ Age at onset of nodding between 3 and 18 years old

$>$ Frequency of nodding 5 to 20 per minute

* Plus at least one of the following minor criteria:

$>$ Other neurological abnormalities (cognitive decline, school dropout due to cognitive/ behavioural problems, other seizures or neurological abnormalities)

$>$ Clustering in space or time with similar cases

$>$ Triggered by food and/or cold weather

$>$ Stunting or wasting

Delayed sexual or physical development

$>$ Psychiatric symptoms.

* A confirmed case: Is a probable case plus a documented nodding episode that is:

$>$ Observed by a trained healthcare worker, or

Videotaped or,

$>$ on EEG/EMG.

These case definitions are to be reviewed and modified as new data becomes available. The same case definitions are applied in this document.

\section{Development of the guidelines}

As part of the Ugandan Ministry of Health clinical response to the disease, in February 2012, the Director General of Health Services constituted a team of nine health workers to develop a manual on treating Nodding Syndrome ${ }^{14}$. This manual aims to guide the training of health workers and provide them with knowledge and skills to manage the patients. The multidisciplinary team comprised of a nurse, health systems and public health specialist, occupational therapist, nutritionist, paediatrician and peadiatric neurologist, physicians and psychiatrists and was later joined by a manager, an obstetrician/ gynaecologist and also received input from a physiotherapist and a speech and language therapist. Initial meetings identified the most important difficulties in individuals with the syndrome and developed a problem list that guided potential interventions. Sources of data included published clinical reports from Tanzania ${ }^{15,16}$ and South Sudan ${ }^{4,}$ ${ }^{17}$, and case investigations by the Ministries of Health, World Health Organization (WHO) and the US Centers of Disease Control (CDC) in Uganda and South Sudan ${ }^{5,18,19}$. Clinical evaluation reports of two sets of patients (8 patients in 2009 and 22 in 2012) brought from Kitgum district to Mulago hospital in Kampala for detailed assessments and better understanding of the syndrome provided a guiding resource for the interventions ${ }^{36}$. Lack of randomised trials for these interventions however underpins the strength of evidence. Lastly, current guidelines for specific disease conditions in the country were adapted as appropriate e.g. in the management of severe malnutrition ${ }^{20,21}$.

\section{Overview}

In the absence of a known cause, a decision was taken to offer symptomatic care. The objective is to relieve symptoms, offer primary and secondary prevention for disability and rehabilitation to improve function. This is a long-term process to be provided by a multidisciplinary team composed of clinicians, nurses, therapists, social workers and teachers. It is suggested that initial management focuses on the most urgent needs of the patient and the immediate family until 'stability' is achieved. The goal is optimal functioning in adulthood. Importantly, health services should be in a stimulating, supporting and caring environment to be able to: Identify the immediate needs of patients;

Offer or refer for inpatient care to manage complications;

Provide outpatient screening and treatment and subsequent follow up care;

Provide psychological and social support for families of children with this chronic and disabling condition; Provide rehabilitation services with occupational, physio and speech and language therapy to improve and restore some of lost functions and prevent further disability;

The most important and urgent needs were considered as seizure control, management of behavioral and psychiatric difficulties, nursing/burns/ wound care, treatment for parasitic infections including microfilaria and helminthes, nutritional and subsequently, physical and cognitive rehabilitation. Other important areas included sexual and reproductive health issues as shown in table 1 . 
Table 1: Main components of the management of patients with Nodding Syndrome Identification and treatment of severe complications

\section{Seizure control}

Management of behavioural, social and psychiatric difficulties

Management of malnutrition

Treatment for parasitic infections including microfilaria and helminthes and vector control

Nursing care

Assessment and rehabilitation of functional difficulties and disabilities

Sexual and reproductive health services

Follow up care Surveillance, documentation, community education and engagement

This management guideline was presented at the first International Conference on Nodding Syndrome in Kampala, Uganda, in July 2012. A hierarchical model to deliver the services was proposed to include emergency care, inpatient, outpatient and community-based care as shown in figure 1.
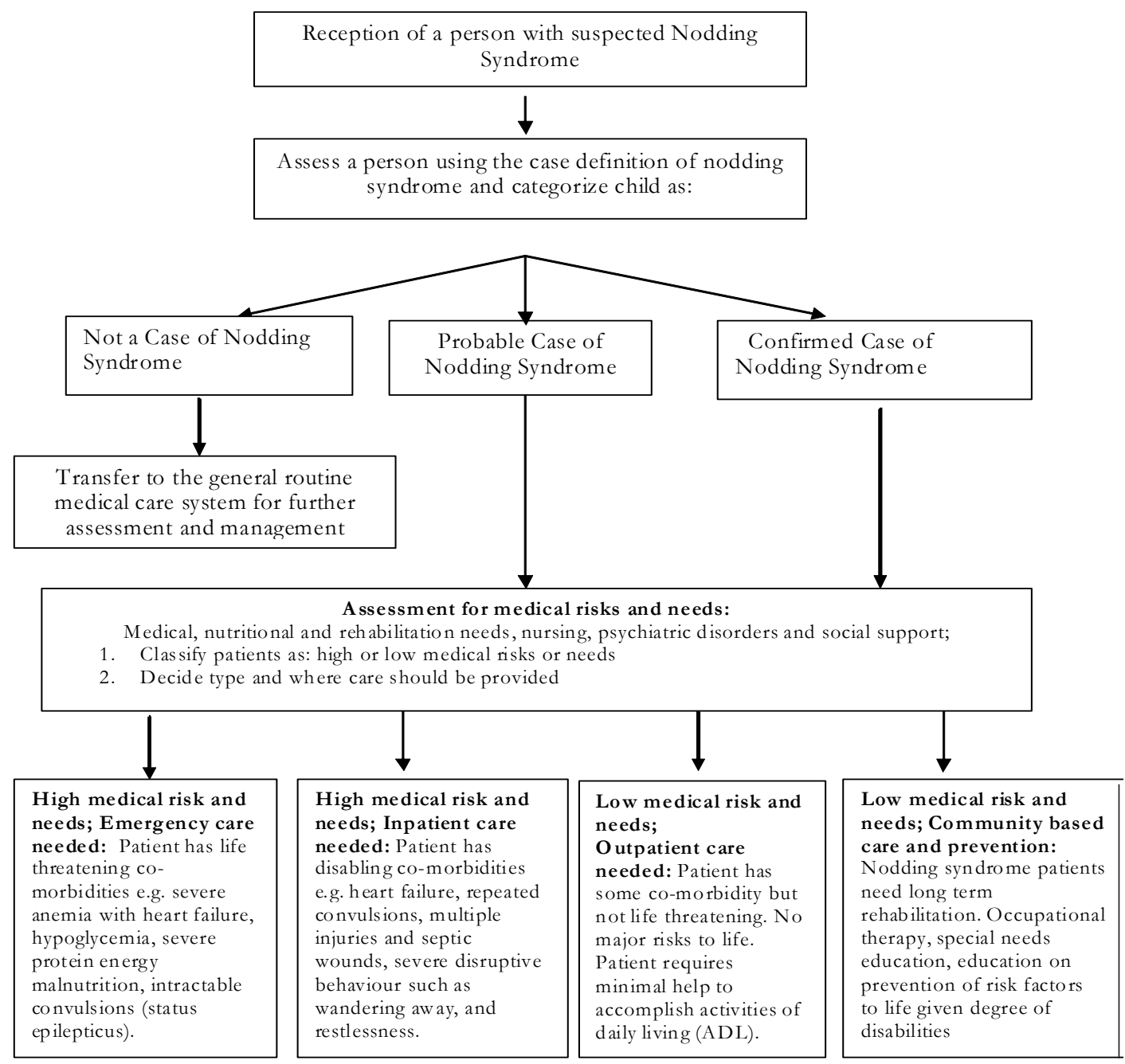

Figure 1: Algorithm for the management of patients with nodding syndrome (Figure adapted from the revised training manual) 


\section{Medical management of Nodding syndrome Emergency care}

Emergency care is indicated for patients with life threatening co-morbidities including status epilepticus, severe anaemia, hypoglycaemia and hypothermia especially in severely malnourished patients, extensive burns and other severe injuries.

i. The initial treatment for status epilepticus should be an intravenous benzodiazepine (Diazepam $0.3 \mathrm{mg} / \mathrm{kg}$, Midazolam $0.3 \mathrm{mg} / \mathrm{kg}$ or Lorazepam $0.1 \mathrm{mg} / \mathrm{kg}$ ). Lorazepam would be the drug of choice but there are difficulties with storage and it is not readily available in many resource limited settings. Subsequent doses of anticonvulsants should follow local protocols for status epilepticus. It should be noted that the risk of status epilepticus may be higher during febrile illnesses, pregnancy and labour.

ii. Severely anaemic patients (haemoglobin $<5 \mathrm{~g} / \mathrm{dl}$ ) with under nutrition should be offered packed blood cell transfusion at $5-7 \mathrm{~mL} / \mathrm{kg}$ if there is concurrent congestive cardiac failure. In the absence of the latter, whole fresh blood transfusion, 10 $\mathrm{mL} / \mathrm{kg}$ should be considered. In either case, transfusion should be slow for over three hours and with frusemide a diuretic.

iii. In children with under nutrition and dehydration, give the rehydration solution for malnutrition (ReSoMal) at $5 \mathrm{~mL} / \mathrm{kg} / \mathrm{hr}$ for the first 2 hours, then $5-10 \mathrm{~mL} / \mathrm{kg}$ ReSoMal alternating with therapeutic milk formula 75 (F75) every hour for up to 10 hours. Any impairment of consciousness should be treated as shock. Shock from dehydration and sepsis are likely to co-exist in patients with severe acute malnutrition. In this case give $10 \%$ glucose at $5 \mathrm{ml} / \mathrm{kg}$ and initiate intravenous antibiotics. Continue fluid therapy with intravenous half-strength Darrow's solution with $5 \%$ glucose, Ringer's lactate with $5 \%$ glucose or $0.45 \%$ (half-normal) saline with $5 \%$ glucose; in all cases, at $15 \mathrm{ml} / \mathrm{kg}$. After two hours, continue rehydration with a nasogastric tube ReSoMal at 5 $-10 \mathrm{~mL} / \mathrm{kg}$ and alternating hourly with F75 for 10 hours $^{20}$.

iv. In the event of extensive burns or other severe injuries, the local protocol for the management of burns and trauma should be applied.

v. Suicidal ideation, attempted suicide and acute severe behaviour problems can occur independently or as a pre-ictal, ictal and post-ictal phases of a seizure. Emergency psychiatric management should ensure safety of both the patient and others. In a severely disturbed child, give diazepam $0.3 \mathrm{mg} / \mathrm{kg}$ IV stat and repeat only once if necessary. Full psychiatric assessment is mandatory when the patient settles. In addition, assess and treat any organic and co-morbid medical condition.

\section{Non emergency inpatient care}

Patients with disabling but non emergency medical conditions and co-morbidities should be managed as inpatients. These may include:

i) Patients with repeated convulsions or a sudden increase in the number of tonic clonic seizures over and above the usual seizure frequency may be at risk of injury or progression to status epilepticus. In these patients, a precipitating event for the increasing seizures (e.g. acute febrile illness, poor adherence, etc.) should be sought and managed. The anticonvulsant drug dose may be titrated upwards. Caution is however advised especially with rapid increases in the dose of anticonvulsants as the pharmacokinetic properties and metabolism of these drugs in patients with Nodding Syndrome especially in the presence of malnutrition are unknown.

ii) Patients with burns requiring admission (e.g. burns of the hands, feet or groin), those with multiple or severe injuries and septic wounds. Wound care with appropriate dressing and debridement if indicated, administration of antibiotics and a booster dose of tetanus toxoid vaccination may be provided. Consideration should also be given for the extra demand of nutrients due to open skin.

iii) Patients with severe and disruptive behaviour such as wandering and restlessness should be managed in conjunction with psychiatry services. Psychiatric assessment should establish reasons for wandering or restlessness.

iv) Patients with other childhood illnesses requiring inpatient care such as pneumonia, diarrhoea with severe dehydration and severe malaria. Management for these conditions should follow usual standards of care for patients without seizures disorders.

Essential investigations include thick and thin blood smears for malaria parasites, blood glucose, complete blood count, peripheral blood film and ESR, blood chemistry for liver and renal function and electrolytes and creatine kinase. In addition, a diagnostic electroencephalogram recording and a skin snip (for a diagnosis of Onchocerca volvulus and Mansonella perstans) 
should be obtained in all patients. Blood cultures, lumbar puncture for cerebrospinal fluid and brain imaging with brain MRI and/or CT scan should be performed as indicated. Lumber puncture is indicated in patients with features suggestive of meningitis or trypanosomiasis. In the case of repeated uncontrolled seizures and maybe new neurological signs/symptoms one should envisage serology for Taenia solium cysticercosis. Skull and limb x rays to exclude fractures from injuries, chest $\mathrm{x}$ rays for evidence of chest infection and $\mathrm{x}$-rays of the wrist to determine bone age may also be performed.

\section{Outpatient care}

Outpatient care is offered to patients with non-life threatening co-morbidity and no major risk to life. This is the standard of care for most patients with Nodding Syndrome. These patients require only minimal help to accomplish activities of daily living. Treatment includes anti epileptic drug therapy, nutritional therapy and rehabilitation with ready to use therapeutic feeds (RUTF), nursing care, cognitive stimulation, physical, occupational and speech and language therapy, and attending to sexual and reproductive issues.

\section{Community based care and prevention}

This is care provided to cater for the long term needs and rehabilitation of patients. It includes provision of physical, occupational and speech and language therapy; adaptive services, return to school and special needs education support, prevention of risk factors for disease and injuries especially in the presence of disabilities. These interventions become even more relevant with improving seizure control and a more ambulant patient. Community education on the syndrome, health promotion services, disease surveillance and monitoring of treatment effects should form important components.

\section{Specific therapies \\ Anti-epileptic drugs}

In Ugandan patients, the main seizure type in Nodding Syndrome, head nodding, has been determined to be an atonic seizure ${ }^{12}$. Other seizure types including absence, tonic clonic and myoclonic seizures may develop 1-3 years after the initial signs. Some patients also develop behaviour and psychiatric difficulties, (Richard Idro, unpublished). In the choice of anti epileptic drugs, we wanted an efficacious, safe and cheap drug against epilepsies with multiple seizure types and with some activity against behavioural and psychiatric difficulties. There are no clinical trials that have examined antiepileptic medications in Nodding Syndrome. Anecdotal reports among patients with head nodding from Tanzania suggest a decrease in seizure frequency when they used phenytoin and Phenobarbitone either individually or in combination ${ }^{16}$. These two are the most common antiepileptic drugs in the affected communities. In developing the guidelines, carbamazepine was not considered because of risk of precipitating myoclonic jerks ${ }^{22}$. Instead, the types of seizures in these patients suggested sodium valproate may be a better option ${ }^{23,24}$ Sodium valproate is also active against some behavioural difficulties like aggression and impulsive behaviour ${ }^{25}$, ${ }^{26}$. It is suggested to start with $10 \mathrm{mg} / \mathrm{kg} /$ day in two divided doses and increase the dose by $5 \mathrm{mg} / \mathrm{kg} /$ day increments until seizure control is achieved or the maximum dose is reached. We suggest a maximum dose of $40 \mathrm{mg} / \mathrm{kg} /$ day in children. Preferably, a single brand should be used consistently as absorption characteristics may differ among brands. The pre-treatment liver function should be determined before initiation of therapy and used in monitoring treatment toxicity.

On each review visit, patients should be assessed for seizure control and for side effects of medications. These include excessive weight gain, liver toxicity (manifesting with deranged liver function, jaundice or abnormal bleeding) and skin rash. The increased risk of fetal malformations with using sodium valproate in females in the reproductive age group may limit its use ${ }^{27}$. It is important to counsel such patients to avoid pregnancy or use second line drugs. Applying these guidelines in a cohort of 22 children studied in Mulago, there was a $57 \%$ reduction in total seizures burden 2-3 weeks after initiation of sodium valproate. Reduction was observed in both clusters of head nodding and number of tonic clonic seizures. The dose range of sodium valproate was then $15-25 \mathrm{mg} / \mathrm{kg} /$ day. We however do not have a recommendation on how long treatment should continue and on weaning (except advise against abrupt cessation of therapy) as it is still early days with use of this treatment regime. This information will possibly be available in future revisions of the guidelines. Additional information on the use of sodium valproate may be found at: http:// www.netdoctor.co.uk/brain-and-nervous-system/ medicines/epilim.html. The suggested second line anti-epileptic drug is lamotrigine. Levetiracetam is also an option ${ }^{28-30}$. To date there is no experience 
with the use of these two drugs in Nodding Syndrome. They may be considered in cases of severe sodium valproate toxicity ${ }^{31}$, failure of response to adequate doses of sodium valproate $-40 \mathrm{mg} /$ $\mathrm{kg} /$ day, pregnancy or girls in the reproductive age group $^{32}$. Lamotrigine is a fairly safe drug but severe skin rashes or reaction may develop. For this reason, the dose should be titrated upwards very slowly. The usual maintenance dose in other childhood epilepsies is $1-10 \mathrm{mg} / \mathrm{kg} /$ day in $1-2$ divided doses and the maximum daily dose is $200 \mathrm{mg}$ in children under 12 years and $400 \mathrm{mg}$ in those 12 years or older. Levetiracetam may be associated with behaviour problems especially in children ${ }^{30,33}$. Anecdotal reports from Tanzania indicate Phenobarbitone and probably phenytoin may be used ${ }^{16}$ (table 1$)$. The benzodiazepines, Clonazepam and Clobazam have been suggested for short term therapy in cases of sharp increases in seizures. Clearly, clinical trials are urgently needed to determine the best treatments

Table 2: Advantages, disadvantages and side effects of suggested antiepileptic drugs for nodding syndrome

\begin{tabular}{|c|c|c|c|c|}
\hline Antiepileptic & Oral Dosing & Advantage & \begin{tabular}{|l} 
Disadvantage \\
\end{tabular} & Main side effects \\
\hline Phenobarbitone & $\begin{array}{l}\text { Usual dose in children } 3-5 \mathrm{mg} / \mathrm{kg} / \mathrm{day} \\
\text { Adolescents }>12 \mathrm{rrs}: 1-3 \mathrm{mg} / \mathrm{kg} / \mathrm{day} \\
\text { in } 1-2 \text { dirided doses. }\end{array}$ & $\begin{array}{l}\text { Cheap and easily } \\
\text { available even in } \\
\text { rural areas } \\
\text { Anecdotal reports } \\
\text { of some success } \\
\text { with tonic clonic } \\
\text { seizures. }\end{array}$ & $\begin{array}{l}\text { Central nerrous } \\
\text { srstem effects and } \\
\text { behaviour } \\
\text { difficulties in } \\
\text { children } \\
\text { There may be no } \\
\text { beneficial effect } \\
\text { on head nodding. }\end{array}$ & $\begin{array}{l}\text { Skin rash. } \\
\text { Drowsiness, lethargy, } \\
\text { unsteadiness, impaired memory } \\
\text { or cognition. } \\
\text { Behariour difficulties }\end{array}$ \\
\hline Phenytoin & $\begin{array}{l}\text { May require a loading dose } \\
\text { Usual dose } 4-8 \mathrm{mg} / \mathrm{kg} / \text { day, max. } \\
\text { Daily dose: } 300 \mathrm{mg} / \text { day. }\end{array}$ & $\begin{array}{l}\text { Cheap and easily } \\
\text { arailable even in } \\
\text { rural areas }\end{array}$ & $\begin{array}{l}\text { A wide range of } \\
\text { side effects. } \\
\text { There may be no } \\
\text { benefit with } \\
\text { seizures in } \\
\text { nodding } \\
\text { sndrome. } \\
\end{array}$ & $\begin{array}{l}\text { Skin rash. Nausea, vomiting, } \\
\text { constipation, drowsiness, } \\
\text { insomnia, nerrousness, tremor, } \\
\text { pins and needles, dizziness, } \\
\text { headache, anorexia, grm } \\
\text { problems, acne, excess hair } \\
\text { growth, and coarse facial } \\
\text { features }\end{array}$ \\
\hline Carbamazepine & $\begin{array}{l}\text { Usual dose } 10-20 \mathrm{mg} / \mathrm{kg} / \mathrm{dar} \\
\text { Start at } 5 \mathrm{mg} / \mathrm{kg} / \mathrm{dar} \\
\text { Dirided } 2 \text { or } 3 \text { times daily Increase } \\
\text { dose ererr week until optimal } \\
\text { response is achiered; maximum } \\
\text { recommended dose: } 35 \mathrm{mg} / \mathrm{kg} / \text { dar. } \\
\text { Berond } 12 \text { rears refer to adult dosing }\end{array}$ & $\begin{array}{l}\text { Available in district } \\
\text { and regional } \\
\text { hospitals in the } \\
\text { affected areas. } \\
\text { Also a mood } \\
\text { stabilizer. } \\
\text { Not recommended }\end{array}$ & $\begin{array}{l}\text { Risk of } \\
\text { precipitating } \\
\text { myoclonic } \\
\text { seizures }\end{array}$ & $\begin{array}{l}\text { Skin rash. } \\
\text { Drt mouth, nausea, romiting, } \\
\text { fluid retention, unsteadiness, } \\
\text { dizziness, drowsiness, fatigue, } \\
\text { and headache, low sodium in } \\
\text { the blood, blood disorders, } \\
\text { dermatitis, and hives. }\end{array}$ \\
\hline Sodium valpoate & $\begin{array}{l}\text { Start with } 10 \mathrm{mg} / \mathrm{kg} / \text { day in two } \\
\text { dirided doses } \\
\text { Increase by } 5-10 \mathrm{mg} / \mathrm{kg} / \text { day weekly } \\
\text { doses in } 2-3 \text { doses untl therapeutic } \\
\text { levels are achiered or up to } \\
40 \mathrm{mg} / \mathrm{kg} / \text { day. }\end{array}$ & $\begin{array}{l}\text { Suggested first line } \\
\text { anti epileptic drug } \\
\text { Broad spectrum } \\
\text { AED } \\
\text { Mood stabilizer }\end{array}$ & $\begin{array}{l}\text { Costly Carries a } \\
\text { higher risk than } \\
\text { other AEDs of } \\
\text { causing fetal } \\
\text { malformations if } \\
\text { taken during } \\
\text { pregnancr. } \\
\text { Pre-conceptual } \\
\text { counselling in } \\
\text { roung girls is time } \\
\text { consuming }\end{array}$ & $\begin{array}{l}\text { Hair loss, nausea, stomach } \\
\text { upset, diarrhoea, and weight } \\
\text { gain and reduced platelets in the } \\
\text { blood. } \\
\text { Polycrstic oraries and menstrual } \\
\text { problems. }\end{array}$ \\
\hline
\end{tabular}


continuation of table 2

An error occurred while printing this page.

Error: rangecheck Offending Command: image

Suggestions:

Value in Postscript file is out of range. Restart your

printer and send document again. Try proof print or

moving some elements off the page. 